INRA Prod. Anim., 2017, $30(5), 479-486$

\title{
Controverses sur la consommation de viande : enseignements d'une analyse sociologique
}

\author{
V. LEGENDRE I, P. SANS ${ }^{2}$, S. BARREY $Y^{3,4}$, B. BOUTIN ${ }^{3,4}$ \\ ${ }_{1}$ IFIP, 31500, Toulouse, France \\ 2 ALISS UR1303, Université de Toulouse, INRA, ENVT, 31076, Toulouse, France \\ ${ }^{3}$ Université de Toulouse, LISST UMR CNRS 5193, 31058, Toulouse, France \\ ${ }_{4}^{4}$ UMR CNRS 5044 - CERTOP, Université de Toulouse, CNRS, UT2J, 31058, Toulouse, France \\ Courriel : p.sans@envt.fr
}

\begin{abstract}
Dans le cadre du Réseau Mixte Technologique «Économie des filières animales », une analyse des controverses sur la consommation de viande a été entreprise, avec l'appui de sociologues de l'Université de Toulouse-Jean Jaurès. Les résultats complètent ceux issus des travaux en cours sur les controverses autour de l'élevage, en particulier ceux initiés par le GIS Élevages Demain (Delanoue et Roguet 2015).
\end{abstract}

La consommation de produits carnés suit ces dernières années une tendance à la baisse. Les viandes bovine, porcine et ovine sont particulièrement concernées, alors que la volaille occupe une place croissante (figure 1).

Parallèlement, les interrogations et les débats sur les produits carnés - appréhendés ici de façon générique et sans distinction par espèce - sont de plus en plus nombreux, engendrant controverses et critiques adressées aux filières : conséquences nutritionnelles, prise en compte du bien-être animal, impacts environnementaux, etc. Ces critiques, portées sur le terrain médiatique, soulèvent la question de leur impact sur les attitudes des consommateurs mais aussi sur leurs comportements d'achat.

Cette contribution a pour objectif de décrire les principaux points de débats qui se posent sur la consommation de viande et d'en déterminer les caractéristiques et les implications. Cette approche ambitionne particulièrement d'identifier les acteurs impliqués dans les controverses, les argumentaires utilisés et comment ils se retrouvent dans l'espace public, ainsi que les évolutions possibles de la consommation de viande ( $"$ mondes possibles »). L'article est structuré de la manière suivante : après une présentation de la méthode utilisée, une analyse des principaux points de débats répertoriés et de leur articulation dans la question plus globale de la consommation de viande (arguments portés, acteurs concernés...) est proposée. Elle débouche sur une cartographie des controverses sur la consommation de viande. Puis, un focus sur un point de débat particulier est proposé : il s'agit de l'abattage des animaux et sa médiatisation, de la publication des premières vidéos par l'association L214 à l'automne 2015 jusqu'à juillet 2016. Enfin, la discussion présente un bilan et dégage les perspectives qu'ouvre ce travail.

\section{1 / Méthode : l'intérêt de la sociologie pragmatique}

Une controverse peut se définir comme « un débat qui engage des connaissances scientifiques ou techniques non stabilisées et qui conduit à des affaires embrouillées, mêlant des considérations juridiques, morales, économiques et sociales. Il s'agit donc d'une situation où les incertitudes usuelles du social, de la politique, de la morale se trouvent compliquées par l'instabilité des connaissances scientifiques ou techniques et l'absence de "faits indiscutables". Si ces controverses ne sont pas limitées au cercle étroit des spécialistes et qu'elles doivent trouver des échos dans l'espace public, elles supposent pourtant toujours des débats autour des connaissances d'ordre scientifique.»1.
Parmi les différentes lectures possibles des controverses, c'est l'approche pragmatiste qui a été retenue (Chateauraynaud et Torny 1999, Callon et al 2001). Elle enseigne que les controverses prennent racine dans une (ou des) incertitude(s) sociotechniques* (les termes affectés d'une astérisque sont définis dans l'encadré 1) - « la consommation de viandes est-elle bonne pour la santé ?» ; « quels sont les impacts de l'élevage sur l'environnement? »- et adoptent des configurations plurielles en fonction des acteurs qui y participent, des alliances qui s'y forment et se défont, des problèmes soulevés et de leurs articulations, de leur dynamique et enfin des solutions proposées par les acteurs impliqués. Dans cette approche, les controverses, loin d'être perçues comme des polémiques inutiles, sont plutôt valorisées dans la mesure où elles ouvrent sur de nouveaux compromis et mondes* possibles (encadré 1). Elle présente donc le double avantage de sortir d'une vision «pathologisante » des controverses (qui considère que toutes les parties prenantes dans une telle situation viennent à manquer de rationalité) et de pouvoir analyser le nouvel ordre social qu'elles instaurent (plutôt que de considérer ces débats comme de simples polémiques stériles, ne faisant que reproduire les rapports de pouvoir existants) (Lemieux 2007).

\footnotetext{
1 Définition utilisée par l'école des Mines-Paristech dans le cadre de son module d'enseignement sur les controverses : http://controverses.mines-paristech.fr/ presentation/quappelons-nous-controverse-sociotechnique/ (consulté le 19 avril 2017).
} 
Figure 1. Évolution de la consommation de viande en France (1960-2015) (Source : IFIP, IDELE, ITAVI).

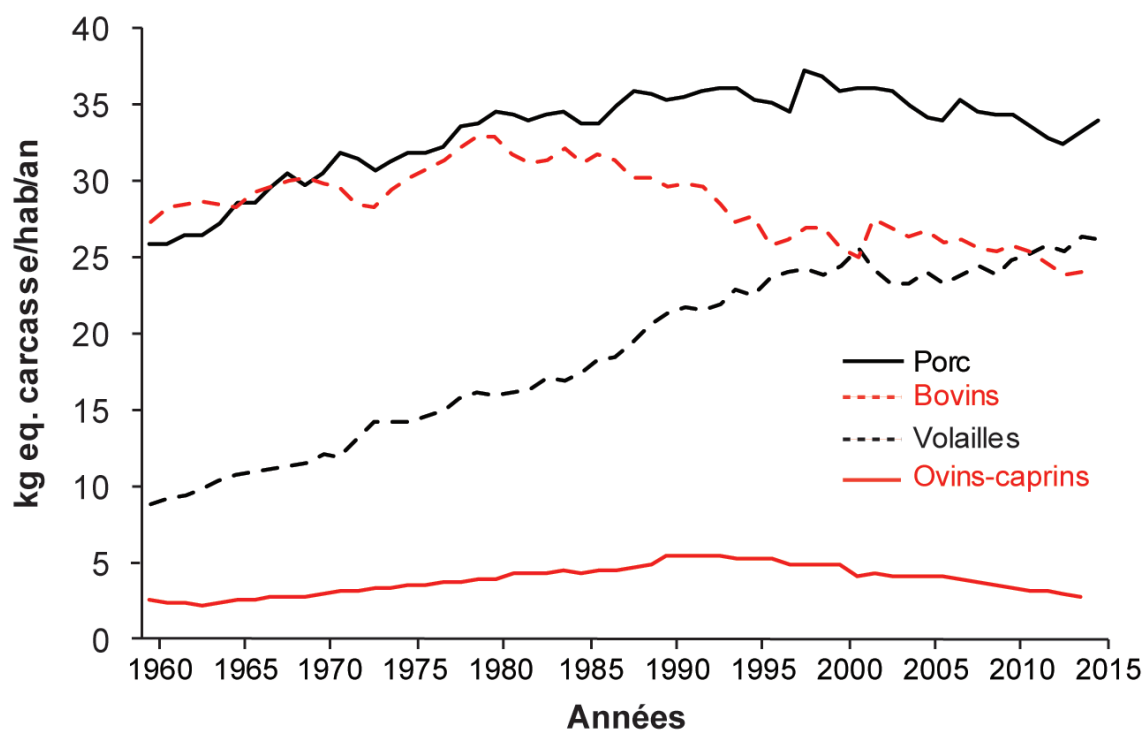

Encadré 1. Définitions.

Cités : Les cités, concept forgé par Boltanski et Thévenot (1991) sont des cadres interprétatifs pour les acteurs impliqués dans des situations de dispute. Elles impliquent des formes d'accords, des objets sociaux différents, qui permettront de reconnaître la nature de la situation, et de savoir sur quel mode de résolution des conflits et des controverses il faut se positionner.

Incertitudes sociotechniques : La notion d'incertitudes a notamment été forgée en économie pour décrire des situations d'asymétrie d'information sur certains marchés (la plupart du temps, il s'agissait d'informations détenues par les vendeurs auxquelles n'avaient pas accès les acheteurs). La notion d'incertitude sociotechnique est mobilisée par les sociologues des sciences et des controverses pour décrire des situations problématiques au sens où les connaissances sur un objet scientifique ou technique (une théorie ou une innovation technique) sont absentes, incomplètes, ou encore débattues dans les champs scientifiques.

Méta-controverse : Une méta-controverse est une controverse dont l'incertitude fondamentale est alimentée par un agglomérat d'incertitudes entretenues par diverses controverses qui semblent interroger un même objet mais au travers de thématisations différentes. Les acteurs qui font vivre les différents débats au sujet de cet objet sont donc les mêmes qui animent la méta-controverse. Certains acteurs se retrouvent à piocher dans des argumentaires exposés dans des débats auxquels ils ne participent pas directement pour renforcer le poids du leur. C'est ce qui rend le lien entre les différentes controverses explicite et qui forme l'unité de la méta-controverse.

Mondes possibles : Contrairement aux cités qui réfèrent à des principes communs déjà constitués et relativement stables, la notion de mondes possibles forgée par Callon et al (2001) fait référence à des mondes toujours mouvants, en devenir, potentiels, en ce sens qu'ils sont des résultats des controverses et non des cadres qui contraignent leur configuration et les épreuves.

Dès lors, l'analyse d'une controverse suppose dans un premier temps l'identification des acteurs qui y prennent part, de la façon dont ils problématisent les produits carnés, et des associations qu'ils tissent avec d'autres (ou au contraire les liens qu'ils rompent) pour faire valoir leur point de vue, en sachant que ces trois éléments sont susceptibles d'évoluer dans le temps. Il s'agit ensuite de dresser un inventaire des problèmes tels les unes aux autres, qui vont servir d'unité d'analyse de la trajectoire et de la dynamique des controverses (Chateauraynaud et Torny 1999);

ii) d'adopter le principe de symétrie, c'est-à-dire de prendre avec le même sérieux les arguments de chaque acteur, dans la mesure où le dénouement de la controverse est incertain ;

iii) de s'intéresser aux processus qui conduisent à l'émergence et à la diffusion des arguments de chacun des acteurs (quels sont les problèmes que la consommation de viande suscite dans l'espace public ?), notamment par le biais du traitement médiatique des prises de position.

Le travail d'analyse a comporté deux étapes (Boutin 2016):

i) Le premier volet a consisté en un large inventaire des points de débats relatifs à la consommation de viande, à partir d'un corpus documentaire (articles de presse généraliste, reportages et débats télévisuels, rapports et études...) issus d'une veille et de la plateforme d'indexation Europresse. L'analyse porte sur les trois dernières années $(2014,2015,2016)$.

Ce travail documentaire a été complété par la réalisation d'une dizaine d'entretiens auprès de responsables professionnels de l'aval des filières (abattage/ transformation, grande distribution et restauration collective), de représentants d'interprofession ou d'organismes de médiation ainsi que de deux consommateurs végétariens. Ces données ont permis de décrire les principaux points débattus et les arguments qu'ils ont suscités, puis de les rendre visibles sur une cartographie. Partant du constat que la plupart des cartographies (représentant les positionnements des acteurs) réalisées en sociologie des controverses sont utiles à l'analyse, mais souvent difficilement lisibles et donc appropriables par les acteurs concernés, nous avons en particulier cherché à en simplifier l'exposition, sans toutefois nuire à l'interprétation des résultats. Cette première étape de l'étude nous permet de considérer que les controverses sur les produits carnés constituent une méta-controverse* (encadré 1) (2 ème partie).

ii) Un second corpus a ensuite été constitué sur la question plus précise de l'abattage des animaux, formé de l'ensemble des journaux télévisés du soir des 4 principales chaînes nationales généralistes gratuites ainsi que des articles de grands quotidiens nationaux de la presse généraliste en utilisant le mot-clé « abattoir ». Les entretiens de la phase exploratoire ont également été mobilisés pour explorer ce thème particulier qui nous conduit à analyser une part impor- 
tante de la construction d'un problème public via sa médiatisation ( $3^{\text {ème }}$ partie).

\section{2 / La consommation de viande, une « méta-contro- verse »}

\section{1 / Quels sont les points débattus et les incertitudes liées à la consom- mation de viande?}

Ces points ont été recensés, décrits et regroupés en quatre thèmes, synthétisés ci-dessous.

\section{a) La santé, thématisée sur des aspects nutritionnels et sanitaires}

Les viandes sont accusées d'être liées à différentes pathologies, en particulier aux cancers, aux maladies cardio-vasculaires, métaboliques ou liées à l'obésité. Par ailleurs, le rôle de la viande comme aliment nécessaire au maintien d'une bonne santé est régulièrement mis en discussion, alors que les alternatives protéiques à base de végétal sont de plus en plus nombreuses. À ces considérations sur la place de la viande dans la nutrition humaine s'ajoutent des interrogations sur les modes de production et la traçabilité. L'usage des antibiotiques de façon systématique est dénoncé car elle nuit à l'efficacité de long terme de ces molécules dans les populations animales voire humaine (antibiorésistance).

\section{b) L'impact environnemental de la pro- duction}

La production de viande est très critiquée pour son impact environnemental. Ces critiques ne sont pas liées à la consommation en tant que telle, mais les arguments touchant à l'environnement sont souvent mis en avant pour justifier la promotion du régime végétarien. Les arguments concernent le rôle de l'élevage dans l'émission de Gaz à Effet de Serre (GES), la pollution de l'eau (problématique de gestion des effluents) et la consommation massive d'eau par l'élevage (en particulier bovin). L'appauvrissement de la biodiversité est également mis en avant, lié à la pollution de l'eau mais également à la sélection génétique qui, en privilégiant des lignées à haut rendement, tendrait à restreindre la diversité génétique.

\section{c) La place des viandes face à la crois- sance démographique}

Les viandes sont également montrées du doigt dans le contexte de la croissance de la population mondiale et de la demande alimentaire. La question porte principalement sur la difficulté à augmenter la production de viande pour faire face à l'augmentation de la population mondiale en tenant compte des niveaux de consommation actuels et donc sur la nécessaire réduction de la consommation. L'accaparement des terres par l'élevage et la concurrence entre ressources animales et végétales sont également mis en avant : il est avancé que les surfaces utilisées pour la production de végétaux entrant dans les rations des animaux pourraient être avantageusement dédiées à l'alimentation humaine.

\section{d) La condition animale}

Enfin, la dernière thématique est celle de la condition animale, très médiatisée au travers de vidéos prises en élevage ou en abattoir. Cette question est transversale, car elle concerne l'élevage des animaux, leur transport et leur abattage. Deux débats transparaissent : d'une part, le statut des animaux dans notre société et la légitimité de tuer pour manger, question centrale dans la position des abolitionnistes; d'autre part, la question du bien-être aux différentes étapes de la vie de l'animal et la capacité du système de production à assurer ce bien-être.

La critique touche plus largement la question de l' « industrialisation de l'élevage », regroupant la problématique de la taille des élevages, des pratiques standardisées visant à une intensification de la production et plus globalement l'ensemble du système sociotechnique de l'élevage (éleveurs intégrés ou, du moins, dépendants d'un système économique qu'ils ne maîtrisent plus...).

Cette mobilisation par les acteurs des arguments issus de plusieurs thématiques, y compris de celles auxquelles ils ne participent pas directement mais qui sont susceptibles de renforcer leur discours, souligne d'une part les appuis argumentatifs qu'ils mobilisent, d'autre part les associations que les acteurs réalisent entre différentes controverses. Ces points d'appuis et ces associations d'idées contribuent à former une méta-controverse sur la consommation de viande.

\section{2 / Trois positions majeures sur la consommation de viande}

Les principaux arguments, les types d'acteurs, leurs objectifs ainsi que les solutions proposées, ont été analysés. Une représentation schématique de la méta-controverse est proposée en figure 2 : cette cartographie rend compte des parties prenantes de la controverse, de leurs arguments et des mondes dans lesquels ils se situent et qu'ils contribuent soit à consolider, soit à promouvoir. Au vu de la diversité des acteurs et de leurs positions, cette figure est une synthèse de la situation sur la scène médiatique, telle que le grand public peut la percevoir.
Elle n'a pas pour ambition de retranscrire l'exhaustivité des points de vue portés par les acteurs, mais de distinguer les grands enjeux qui sous-tendent la controverse et comment ils s'articulent. Les frontières entre les positions des différents protagonistes sont ténues et les points de vue des acteurs d'une même catégorie peuvent diverger.

Trois positions principales ont été distinguées sur la consommation de viande :

\section{a) Une position "végétarienne "}

Elle est défendue par des associations abolitionnistes comme L214 ou environnementalistes et alimentée par des rapports émanant d'organisations internationales, comme par exemple le rapport Livestock's Long Shadow de la FAO (2006), qui soulignent - entre autres - la contribution de l'élevage de ruminants à la production de GES. Les défenseurs de cette position envisagent un monde où l'élevage serait aboli et où les régimes alimentaires excluraient tous les produits animaux, en y substituant des végétaux.

b) Une position prônant une consommation alternative de viande (" consommer mieux»)

Elle est notamment portée par des associations défendant le respect de l'environnement et du bien-être animal, comme les ONG « welfaristes » CIWF et Welfarm ou des professionnels des filières (notamment au sein du collectif Quand l'Abattoir Vient à la Ferme). Les critiques adressées aux filières animales ne sont pas liées à l'acte de production lui-même, mais à son caractère " industriel ». La solution défendue n'est pas d'y mettre fin mais de le réformer en mettant en avant des systèmes alternatifs renforçant le lien social. La perspective envisagée est de consommer mieux, de manière plus qualitative, quitte à consommer moins.

c) Une position visant au maintien de la place de la consommation de viande et au développement de l'activité des filières animales

Elle est défendue notamment par les professionnels des filières et les organismes qui les représentent. Les réponses aux critiques adressées aux filières sont essentiellement technico-organisationnelles : les solutions passent à la fois par des innovations techniques (optimisation des rations alimentaires pour diminuer la production de GES...), des innovations dans les systèmes d'élevage (réduction des intrants dont les antibiotiques, meilleure prise en compte du bien-être animal...) et par des interrelations entre les maillons des filières (contractualisation). 
Figure 2. Représentation schématique et synthétique de la méta-controverse sur la consommation de viande : arguments, acteurs, objectifs.

Les codes-couleur des arguments sont indiqués en rappel dans les cases présentant les acteurs.

UNEP : « United Nations Environment Programme »; FAO : « Food and Agriculture Organization » ; GIEC: Groupe Intergouvernemental sur l'Evolution du Climat ; OMS : Organisation Mondiale de la Santé ; CFBCT : Confédération Française de la Boucherie, Boucherie-Charcuterie, Traiteurs ; Collectif QAVF : Quand l'Abattoir Vient à la Ferme ; CIV : Centre d'Information sur les Viandes ; INRA : Institut National de la Recherche Agronomique.

Lecture : les environnementalistes mobilisent des arguments issus des pôles « Protéger l'environnement », « Nourrir les générations futures » et "Protéger les animaux ».

\section{Arguments}
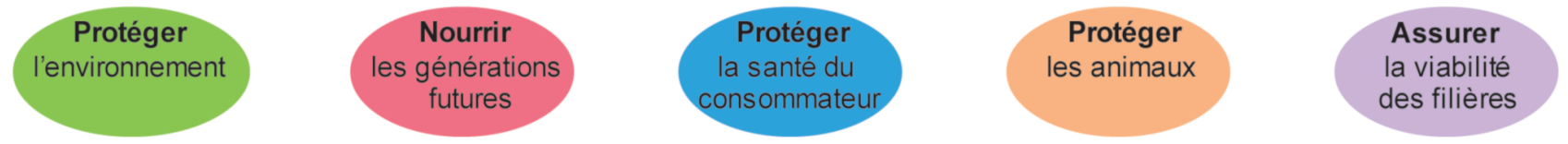

\section{Acteurs}

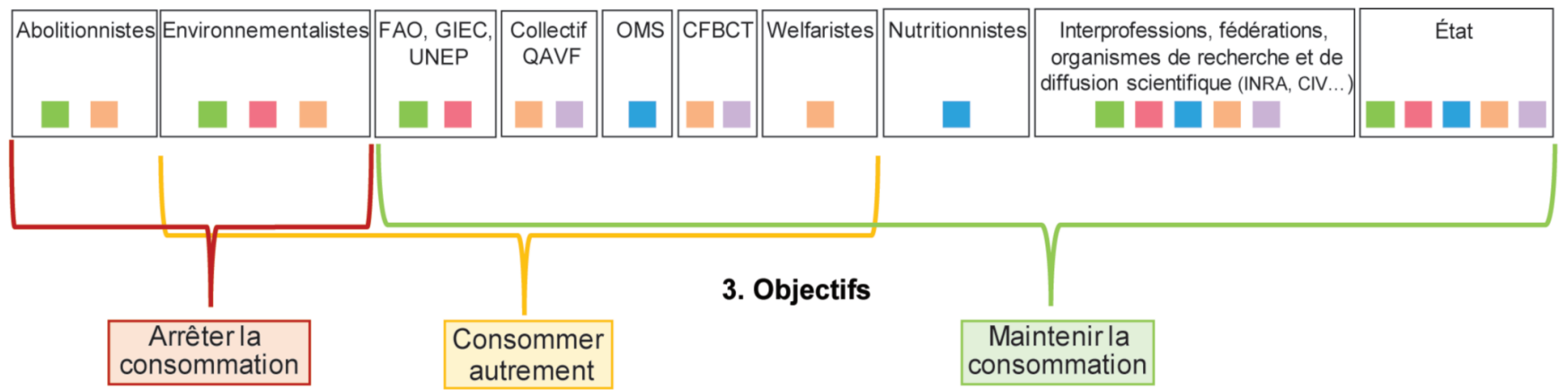

\section{3 / Différents cadres interpré- tatifs au cour des débats}

Les « cadres interprétatifs » constituent, dans la théorie développée par Boltanski et Thévenot (1991), des cadres de références mis au point et utilisés par les personnes afin de procéder à l'évaluation de situations d'action et à prendre des décisions. Ils cadrent des logiques de justification (dans une dispute) pour orienter les débats et fixer le sens de ce qui est « juste » et de ce qui ne l'est pas. Ils impliquent des formes d'accord, des entités plus ou moins valorisées selon les différents cadres (les animaux, les éleveurs ou l'environnement par exemple), qui permettront de définir les problèmes et leur mode de résolution. Dans une dispute, le moment crucial est celui de l'épreuve, c'est-à-dire le moment où des acteurs (ou groupes d'acteurs) s'affrontent en se référant à des « principes supérieurs communs » : en situation de dispute publique, les acteurs ne revendiquent pas ce qu'ils désirent au nom d'intérêts privés (qui ont peu de chances d'être pris au sérieux par les opposants), mais au nom d'une conception de ce qui est juste pour tous, et universalisable, dans cette circonstance.

Ainsi, une même question peut être envisagée de manière très différente selon le cadre interprétatif mobilisé. Boltanski et Thévenot ont recensé les grands « prin- cipes », qui valent dans le cadre de la culture de l'Europe occidentale et, en particulier, de la France et sont le support de six cités* (encadré 1) : civique, industrielle, domestique, de l'opinion, inspirée et marchande. Ces cités ont été complétées par d'autres cadres interprétatifs dans la littérature sociologique, notamment par la cité « verte » correspondant à la cité écologique.

Pour notre analyse, ces cités dans lesquelles sont promus des principes supérieurs communs sont heuristiques dans la mesure où elles permettent de repérer des moments où les acteurs ne sont pas d'accord sur les principes supérieurs communs à défendre, ou encore des moments où différents acteurs se réfèrent au même bien commun mais ne sont pas d'accord sur qui a droit à quoi, en fonction de ce principe. Elles permettent aussi d'envisager des transformations des rapports de force et des évolutions à plus ou moins long terme des principes supérieurs communs.

Ainsi de notre analyse, trois cadres interprétatifs distincts émergent : " antispéciste », « paysan » et « industriel », qui peuvent être reliés à trois des cités définies par Boltanski et Thévenot (tableau 1).

Dans le cadre antispéciste, l'humain est un animal comme les autres. L'élevage est perçu comme un système d'exploita- tion d'êtres vivants par d'autres êtres vivants et les abattoirs représentent des lieux d'exécutions massives. Dans ce cadre, consommer de la viande, c'est cautionner une forme d'esclavagisme et participer à un génocide. Ce cadre partage certaines caractéristiques de la cité « civique (Boltanski et Thévenot 1991): la communauté englobe tous les êtres vivants et les valeurs prônées sont celles de l'équivalence entre les êtres concernées.

Dans le cadre paysan, l'homme fait partie intégrante de son écosystème. L'humain et l'animal n'entretiennent pas une relation utilitaire, mais un échange de dons et de contre dons suivant le principe de la réciprocité. L'homme soigne l'animal et le nourrit, en garantissant son bien-être, en échange des produits que l'animal lui fournit. La consommation de viande y est donc un acte raisonné, porteur de sens. Ce cadre partage avec la « cité verte », la dénonciation $d u$ mode de production industriel et du productivisme (Lecourt 1992).

Le cadre industriel appuie son développement sur les révolutions agraires de l'après-guerre et sur les décisions de modernisation de l'agriculture (Hervieu et Purseigle 2013). Les différents maillons des filières animales se sont équipés de moyens les rendant toujours plus performants. La parole des scientifiques et des 
Tableau 1. Caractéristiques des différents cadres interprétatifs.

\begin{tabular}{|l|c|c|c|}
\hline Cadre interprétatif & Antispéciste & Paysan & Industriel \\
\hline Cité & Civique & Verte & La société humaine \\
\hline $\begin{array}{l}\text { Communautrielle de } \\
\text { référence }\end{array}$ & Le règne animal & $\begin{array}{c}\text { L'ensemble des composantes } \\
\text { de l'écosystème }\end{array}$ & Le qui protège l'écosystème \\
\hline $\begin{array}{l}\text { Caractéristiques } \\
\text { valorisées }\end{array}$ & $\begin{array}{c}\text { L'égalité, l'intérêt général } \\
\text { techniques, le progrès scientifique }\end{array}$ \\
\hline $\begin{array}{l}\text { Caractéristiques } \\
\text { dévalorisées }\end{array}$ & $\begin{array}{c}\text { Les intérêts particuliers } \\
\text { des humains }\end{array}$ & $\begin{array}{r}\text { Ce qui le pollue, ce qui le } \\
\text { détruit }\end{array}$ & La méconnaissance, le profane \\
\hline
\end{tabular}

experts, basée sur les résultats chiffrés qu'ils produisent, sont valorisés, à l'inverse de celle des profanes. La consommation de viande, désormais économiquement accessible à tous, y est considérée comme un progrès permis par la performance des moyens de production.

Si les cités sont heuristiques pour l'analyse, la théorie de la justification de Boltanski et Thévenot présente toutefois certaines limites. La plus importante est liée au postulat que ces moments de dispute suspendent pendant un temps l'action. Or, dans la méta-controverse sur la consommation de viande, ces trois cadres interprétatifs évoluent en interaction au cours des actions entreprises par les parties prenantes. En outre, ce n'est pas l'identité professionnelle ou profane ou encore citoyenne des personnes qui détermine le cadre interprétatif qui leur sert de référence. Selon les situations qui s'enchaînent très rapidement dans le cas de cette méta-controverse, les acteurs peuvent se positionner dans différents mondes. Par exemple, lorsqu'une association environnementaliste dénonce la consommation de viande en avançant des données chiffrées sur les quantités d'eau nécessaires à la production des animaux, elle se positionne dans le monde industriel, en valorisant une expertise et des données chiffrées.

Pour qu'une controverse puisse se clôturer (au moins pour un temps), il est nécessaire que les acteurs partagent un cadre interprétatif suffisamment homogène et stable. Cela donne lieu à des rapports de force, chaque acteur tentant d'imposer aux autres ses principes supérieurs. Aujourd'hui le cadre industriel et ses principes apparaissent comme le cadre dominant. Les arguments environnementalistes et antispécistes s'efforcent de se baser sur des résultats scientifiques pour gagner en robustesse sur les questions d'abattage, de transport et d'amélioration des techniques d'élevage en lien avec le bien-être animal. Mais le renforcement de la place des antispécistes tend toutefois à déplacer la controverse autour d'arguments basés sur l'égalité entre humains et animaux. La diffusion accrue du discours des antispécistes par les médias matérialise ce déplacement, notamment sur la question de l'abattage des animaux. L'effet de ce déplacement sur les comportements de consommation réels n'est pas quantifiable à l'heure actuelle.

\section{3 / Médiatisation de la ques- tion de l'abattage}

L'abattage des animaux est particulièrement présent dans les médias depuis la diffusion de vidéos « choc » par l'association L214 à partir de l'automne 2015. Cette question et la façon dont elle est médiatisée ont fait l'objet d'une analyse plus approfondie. Les journalistes jouent en effet un rôle particulier dans la mise à disposition de l'information sur le sujet, agissant comme un filtre entre l'émetteur de l'information et le récepteur (le grand public).

\section{1 / Différentes façons de poser le problème}

Selon les différents cadres interprétatifs qui cohabitent et à travers lesquels les acteurs évoluent, la question des conditions d'abattage des animaux, montrée par les vidéos, est problématisée de manière différente. Chaque cité définit sa manière d'aborder le problème.

Dans le cadre antispéciste, le problème vient du fait de tuer des êtres capables de souffrir, dotés de sentiments et d'émotions. L'abattage des animaux n'est qu'une partie du problème, puisque c'est l'exploitation animale dans son ensemble que les antispécistes veulent abolir. Ils sont partisans d'une rupture radicale avec le régime alimentaire actuel et visent à l'arrêt de la consommation de viande.

Dans le cadre industriel, le problème vient de la méconnaissance qu'ont les consommateurs de l'étape d'abattage, nécessaire à la production de viande, qui renvoie au rapport de chacun à la mort. Plus largement, les professionnels observent une déconnexion des consomma- teurs avec les pratiques de production qui génère un manque de confiance. L'allongement des circuits de commercialisation et l'urbanisation croissante de la population conduisent à une rupture des liens avec les mondes agricole et agroalimentaire. Enfin, si le manque de moyens financiers des abatteurs face aux obligations réglementaires peut entraîner des situations anormales, les cas de maltraitance animale restent isolés et ne sont pas généralisables à l'ensemble du secteur. Pour les tenants du cadre industriel, il est nécessaire d'investir pour promouvoir l'émergence et la diffusion de modèles innovants (en termes d'impact environnemental ou d'amélioration du bienêtre animal). En outre, un effort particulier doit porter sur la formation professionnelle et la responsabilisation des agents ainsi que sur les moyens de contrôle (inspecteurs de santé publique vétérinaire en abattoirs, par exemple).

Dans le cadre paysan, le problème vient de la perte de lien entre les éleveurs, considérés comme garants du bien-être animal, et les opérateurs industriels. Les promoteurs de ce modèle militent pour une transition vers un autre modèle agricole et alimentaire, privilégiant des ateliers de production à taille humaine, des outils d'abattage de plus petite taille (voire l'abattage à la ferme) et une plus grande proximité géographique et humaine entre les acteurs des filières.

Ces différentes problématisations entrent en concurrence sur le terrain médiatique pour tenter de s'imposer auprès de l'opinion publique comme la « vraie » définition du problème. Les journalistes jouent un rôle clef. L'orientation de leurs articles et le choix des acteurs qui s'expriment ont contribué à l'émergence d'une problématisation dominante, où certains faits sont plus saillants que d'autres.

\section{2 / Une problématisation domi- nante et une logique accusatoire}

À l'automne 2015, les premières communications de L214 (visant notamment 
l'abattoir d'Alès) dénonçaient les mauvaises conditions d'abattage en mettant en avant des aspects « techniques » (manquements aux réglementations en termes d'étourdissement et d'hygiène, manque de formation des salariés, faible présence des services vétérinaires...) et leurs conséquences en termes de risques sanitaires. Puis c'est rapidement la souffrance animale, qui va de pair avec la sensibilité attribuée aux animaux dans la logique des antispécistes, qui va s'imposer comme l'épicentre du problème. Le lien avec la consommation est mis en avant, notamment par la mise en cause de la légitimité de tuer les animaux pour se nourrir.

La multiplication des vidéos, montrant notamment que le problème touche également des abattoirs impliqués dans des filières sous signes de qualité, et le renforcement des arguments antispécistes vise à généraliser le problème en considérant l'ensemble du secteur de l'abattage comme une « boîte noire » (généralisation horizontale). À l'inverse, les professionnels de l'abattage arguent de responsabilités individuelles, ce qui circonscrit le problème aux abattoirs incriminés. Accusés d'être responsables de cette situation en raison d'un déficit de contrôle, les pouvoirs publics condamnent ces actes de maltraitance. Au terme de ce processus, la question de l'abattage et de la souffrance animale est finalement inscrite à l'agenda public, au travers de la mise en place d'une commission parlementaire chargée d'élaborer une proposition de loi contenant des mesures de prévention et de contrôle. La proposition de loi a été adoptée en janvier 2017. La reconnaissance du problème comme " public " montre que le phénomène de généralisation horizontale a fonctionné et conforte l'action des abolitionnistes.

Au cours du processus de médiatisation de la question de l'abattage des animaux, les journalistes ont tendance à donner aux informations qu'ils traitent une forme accusatoire, à travers la désignation des victimes, des dénonciateurs, des persécuteurs et des juges.

Dans la problématisation dominante, les militants antispécistes, des personnalités se posant comme porte-paroles de la cause et une partie des journalistes vont occuper la position de dénonciateurs, en défendant les animaux qui sont alors perçus comme des victimes. Le secteur de l'abattage-découpe et plus largement les filières de production de viande (éleveurs, transformateurs), qu'elles soient conventionnelles ou alternatives (labels de qualité, agriculture biologique...) se voient attribuer le rôle de persécuteurs. Ce sont plutôt le « système de production de viande » ou le « système d'abat- tage », perçus comme des entités désincarnées, qui sont accusés. Quant à l'État et ses différents représentants (services publics), ils sont à la fois acteurs du problème et juges. L'implication directe de la consommation de viande pose également les consommateurs comme juges. Leur comportement d'achat les positionne aussi comme acteurs, sur le mode « vous avez le pouvoir de faire changer les choses $»$.

\section{3 / Une évolution de la place des différents cadres interprétatifs}

Au travers de la stigmatisation de l'abattage et de la mise en cause de la consommation de viande, le cadre antispéciste et ses militants affichent une ambition bien plus large : celle de mettre fin à toute forme d'exploitation animale et de promouvoir le véganisme. Leur implication active sur la condition animale à l'abattoir constitue un angle d'attaque opportuniste sur une question qu'ils ont contribué à rendre médiatique et sensible pour l'opinion publique. Leur position et leur visibilité dans la controverse sur la consommation de la viande s'est renforcée. La multiplication des contacts entre militants antispécistes et journalistes peut générer un phénomène d'enrôlement (Callon 1986) de ces derniers en faveur de la diminution ou de l'arrêt de la consommation de viande, susceptible de faire évoluer la méta-controverse.

À l'inverse, le cadre industriel s'est affaibli, dans un contexte de recul sensible de la consommation et de préoccupation croissante des consommateurs. Le bien-être animal apparaît comme « l'inquiétude » principale des français face à la viande (OCHA 2016).

En parallèle, au sein du cadre paysan s'est développé un argumentaire dénonçant des cadences d'abattage intenables pour les opérateurs et une industrialisation trop poussée et prônant des méthodes alternatives et une consommation plus qualitative.

\section{4 / Bilan, discussion et per- spectives}

La méta-controverse sur la consommation de viande apparaît dominée par des questions liées à la production, plus qu'à la consommation elle-même. L'environnement, le bien-être animal et plus largement les modes de production font partie des questions centrales pour le grand public. Les questions nutritionnelles, bien que de plus en plus prégnantes dans le débat, sont encore souvent cantonnées à la sphère scientifique. Les questions paraissent porter principalement sur l'élevage, bien que la médiati- sation de l'abattage déplace également le débat vers les maillons plus en aval. Cela a pour effet de mettre en lumière la mort animale, un monde inconnu qui n'est agréable pour personne. Par ailleurs, la place croissante dans les modes de consommation contemporains de produits de plus en plus élaborés, en substitution des produits carnés bruts, tend à distendre le lien entre consommation et élevage.

La représentation schématique de la méta-controverse ne permet pas de rendre compte de son évolution dynamique. L'évolution des débats modifie la structure de la controverse et de ses acteurs. Les transformations sociales entraînent des partenariats et des alliances entre les différents protagonistes, notamment entre professionnels des filières et associations pour construire des cahiers des charges pour une production plus respectueuse des animaux et de l'environnement. Par ailleurs, l'essor des questions sur le bienêtre animal a engendré la création d'une commission " enjeux sociétaux » au sein de l'interprofession Interbev.

Le rapport de force entre les cadres interprétatifs s'est toutefois durci, notamment entre industriel et antispéciste, rendant actuellement impossible un dialogue constructif sur un terrain de discussion commun, condition nécessaire à l'avancée d'une controverse vers sa clôture.

Sur un sujet aussi vaste que la consommation de viande et dans un temps de réalisation relativement court, les points de débats recensés et la description de la méta-controverse ne peuvent prétendre à l'exhaustivité. Certains constats interpellent cependant :

i) peu de nuances sur la situation des différentes viandes apparaissent dans l'analyse des corpus, en particulier du point de vue nutritionnel.

Ce critère joue cependant un rôle important dans l'évolution de la consommation des différentes espèces, bénéficiant aux viandes blanches au détriment des viandes rouges. À titre d'exemple, plusieurs débats sont apparus dans les médias ces derniers mois (rapport de l'OMS liant viande rouge et cancer fin octobre 2015, polémiques sur l'utilisation de sel nitrité dans les charcuteries en septembre 2016, recommandations de l'ANSES incitant à réduire viande rouge et charcuterie en janvier 2017...).

ii) Certains acteurs semblent relativement peu présents dans le débat, notamment la sphère de recherche et développement proche des filières animales (chercheurs, scientifiques, instituts techniques...). 
Les critiques adressées aux filières animales font toutefois l'objet de réponses argumentées par des scientifiques (Laporte et Mainsant 2012, Rosner et al 2016) tandis que les conséquences néfastes du régime végétarien sont également montrées du doigt (Legrand 2015).

iii) La mise en accusation, dans la problématisation dominante, du « système de production » comme une entité désincarnée médiatise peu la place des hommes.

Aux différents points de tension recensés pourrait se rajouter la dimension sociale en élevage et en abattoir, notamment la condition des travailleurs de l'industrie de l'abattage-découpe, stigmatisée par un livre début 2017 (Le Guilcher 2017).

\section{Références}

Boltanski L., Thévenot L., 1991. De la justification. Les économies de la grandeur, Gallimard Paris, France, 496p.

Boutin B., 2016. Le monde d'une méta-controverse : consommer de la viande ? Mémoire de Master 2, Université Toulouse Jean Jaurès, Décembre 2016, 77p.

Callon M., 1986. Éléments pour une sociologie de la traduction. La domestication des coquilles Saint-Jacques et des marins dans la baie de SaintBrieuc, L'année sociologique, 36, 170-208.

Callon M., Lascoumes P., Barthe Y., 2001. Agir dans un monde incertain : Essai sur la démocratie technique, Édition du Seuil, Paris, France, $368 \mathrm{p}$.

Chateauraynaud F., Torny D., 1999. Les sombres précurseurs : Une sociologie pragmatique de
Dans notre analyse, le recueil d'informations a privilégié des médias « classiques » (journaux, chaînes de télévision). La place des réseaux sociaux et leur rôle dans la transmission d'informations n'ont pas été pris en compte. Or, ces canaux prennent une importance considérable, tant dans la stratégie des acteurs que dans les pratiques des individus. Les mondes et cités sont présentés ici tels des photographies à un instant donné. Or, ces mondes et leurs caractéristiques évoluent, sous l'effet des arguments échangés et des « coups » portés.

Des approfondissements seraient nécessaires pour préciser les positions prises par les différents acteurs. En exploitant un corpus documentaire essentiellement constitué de données destinées au grand public, le travail ambitionne de refléter les informations accessibles aux citoyens français, telles qu'elles sont relayées par les médias.

Les réactions des consommateurs face aux différentes composantes de la controverse nécessitent d'être approfondies, alors que les modes de consommation évoluent et que les déterminants sont multiples. La médiatisation de sujets polémiques tels que l'abattage des animaux suscite de vives réactions (commentaires en réponse aux articles publiés sur internet, messages aux associations de consommateurs...) mais comment influent-elles sur le comportement et le choix des consommateurs face aux produits carnés ? l'alerte et du risque, Éditions de l'EHESS, Paris, France, $476 \mathrm{p}$

Delanoue E., Roguet C., 2015. Acceptabilité sociale de l'élevage en France : recensement et analyse des principales controverses à partir des regards croisés de différents acteurs, INRA Prod. Anim., 28, 39-50.

FAO, 2006. Livestock's long shadow: environmental issues and options, Rome, Italie, 416p.

Hervieu B., Purseigle F., 2013. Sociologie des mondes agricoles, Armand Colin, Paris, France, $318 \mathrm{p}$.

Laporte R., Mainsant P., 2012. La viande voit rouge, Éd. Fayard, Paris, France, 224p.

Lecourt D., 1992. Le paradoxe de l'environnement. SRETIE Infos, 37.
Le Guilcher G., 2017. Steak machine, Éd. Goutte d'or, Paris, France, 170p.

Legrand P., 2015. Coup de pied dans le plat, Éd. Marabout, Paris, France, 256p.

Lemieux C., 2007. À quoi sert l'analyse des controverses? Mil neuf cent, 25, 191-212.

OCHA-CERTOP-CREDOC, 2016. Synthèse des résultats de l'étude sur les inquiétudes alimentaires, $17 \mathrm{p}$

Rosner P.M., Hocquette J.F., Peyraud J.L., 2016. Peut-on encore légitimement manger de la viande aujourd'hui? Revue de l'Académie d'Agriculture, 8, 32-34.

\section{Résumé}

La consommation de produits carnés fait l'objet de débats parfois très vifs, dans un contexte de décroissance globale des quantités ingérées par habitant. Cette contribution a pour objectif d'identifier les principales interrogations des acteurs des filières animales et celles de leurs détracteurs sur les produits carnés, et de cerner les potentiels points de débats qui peuvent en résulter. En adoptant l'approche de la sociologie pragmatiste, les acteurs impliqués dans les controverses sont repérés ainsi que les argumentaires utilisés et la façon dont ils se retrouvent dans l'espace public. Un focus particulier est porté sur la question de l'abattage des animaux et sa médiatisation, depuis la publication des premières vidéos par l'association L214 (automne 2015) jusqu'à juillet 2016. La métacontroverse sur la consommation de viande apparaît dominée par des questions liées à la production, plus qu'à la consommation ellemême. L'environnement, le bien-être animal et plus largement les modes de production font partie des questions centrales pour le grand public. Les questions nutritionnelles, bien que de plus en plus prégnantes dans le débat, sont encore souvent cantonnées à la sphère scientifique. Les questions paraissent porter principalement sur l'élevage, bien que la médiatisation de l'abattage déplace également le débat vers les maillons plus en aval. Elles traduisent l'existence de trois positions vis-à-vis de la consommation de viande (végétarienne, alternative et maintien) qui renvoient à trois cadres interprétatifs (antispéciste, paysan et industriel). L'évolution des attitudes des consommateurs et la place relative de chacune de ces trois positions constituent des facteurs susceptibles d'influencer les volumes et types de produits carnés consommés dans les années à venir. 


\begin{abstract}
Controversies on meat consumption : lessons from a sociological perspective

Meat product consumption is a much-debated topic. This contribution aims at identifying the major hot points and determining their characteristics and their implications. By adopting the point of view of pragmatic sociology, players involved in the controversies are identified, as well as the cases used and the way in which they become a public concern. Special attention is paid to the question of animal slaughtering and its media coverage, since the launching of the first videos by the association L214 (autumn 2015) until July 2016. The meta-controversy on meat appears dominated by questions linked with production, more than with its consumption. Environment, animal welfare and more largely production systems are part of the key questions for opinion. Even if the nutritional topics are more and more pregnant in the debate, they seem to be confined to the scientific community. Citizens' concerns appear to relate mainly to breeding, even if the media coverage of slaughtering partially transfer the debate to the downstream of the supply chain. They illustrate the existence of three positions with respect to meat consumption (veggie, alternative and status-quo) which refer in three worlds (antispecist, peasant and industrialist). Changes in consumers' attitudes and the respective importance of each of these three points of view are likely to influence the volumes and types of products sold, in the future.
\end{abstract}

LEGENDRE V., SANS P., BARREY S., BOUTIN B., 2017. Controverses sur la consommation de viande : enseignements d'une analyse sociologique. INRA Prod. Anim., 30, 479-486. 